\title{
Risks and Benefits of Prophylactic Inferior Vena Cava Filters in Patients Undergoing Bariatric Surgery
}

\author{
Nancy J. Birkmeyer, PhD, Jonathan F. Finks, MD¹, Wayne J. English, MD², Arthur M. Carlin, MD³, \\ Abdelkader A. Hawasli, MD, FASMBS ${ }^{4}$, Jeffrey A. Genaw, MD³, Michael H. Wood, MD , David A. Share, MD, \\ John D. Birkmeyer, MD1, for the Michigan Bariatric Surgery Collaborative
}

\begin{abstract}
${ }^{1}$ Center for Healthcare Outcomes and Policy and Department of Surgery, University of Michigan, Ann Arbor, Michigan; ${ }^{2}$ Surgical Weight Loss Center, Marquette General Hospital, Marquette, Michigan; ${ }^{3}$ Department of Surgery, Henry Ford Hospital, Detroit, Michigan; ${ }^{4}$ Department of Surgery, St. John Hospital and Medical Center, Detroit, Michigan; ${ }^{5}$ Department of Surgery, Detroit Medical Center, Detroit, Michigan; ${ }^{6}$ Value Partnerships Program, Blue Cross and Blue Shield of Michigan, Detroit, Michigan.
\end{abstract}

BACKGROUND: The United States Food and Drug Administration recently issued a warning about adverse events in patients receiving inferior vena cava (IVC) filters.

OBJECTIVE: To assess relationships between IVC filter insertion and complications while controlling for differences in baseline patient characteristics and medical venous thromboembolism prophylaxis.

DESIGN: Propensity-matched cohort study.

SETTING: The prospective, statewide, clinical registry of the Michigan Bariatric Surgery Collaborative.

PATIENTS: Bariatric surgery patients $(n=35,477)$ from 32 hospitals during the years 2006 through 2012.

INTERVENTION: Prophylactic IVC filter insertion.

MEASUREMENTS: Outcomes included the occurrence of complications (pulmonary embolism, deep vein thrombosis, and overall combined rates of complications by severity) within 30 days of bariatric surgery.

The use of inferior vena cava (IVC) filters has increased substantially in recent years. These medical devices, which are used to prevent pulmonary embolism in patients considered to be at high risk of venous thromboembolism, were placed in 167,000 patients in 2007. ${ }^{1}$ In 2012, it is estimated that 259,000 patients will undergo placement of an IVC filter, an increase of $55 \%{ }^{1}$ Increasing use of IVC filters is attributable to the development of retrievable versions of the devices, which have expanded indications for use such as in bariatric surgery.

Unfortunately, the increase in the use of IVC filters has been accompanied by an increase in reports of

*Address for correspondence and reprint requests: Nancy J. Birkmeyer, PhD, Center for Healthcare Outcomes and Policy, University of Michigan, Room 140E, Bldg. 16, North Campus Research Complex, 2800 Plymouth Road, Ann Arbor, Ml 48109; Telephone: 734-998-7472;

Fax: 734-998-7473; E-mail: nbirkmey@umich.edu

Additional Supporting Information may be found in the online version of this article.

Received: November 7, 2012; Revised: December 18, 2012; Accepted: December 23, 2012

2013 Society of Hospital Medicine DOI 10.1002/jhm.2013

Published online in Wiley Online Library (Wileyonlinelibrary.com).
RESULTS: There were no significant differences in baseline characteristics among the 1,077 patients with IVC filters and in 1,077 matched control patients. Patients receiving IVC filters had higher rates of pulmonary embolism $(0.84 \%$ vs $0.46 \%$; odds ratio [OR], 2.0; 95\% confidence interval [Cl], 0.6$6.5 ; P=0.232)$, deep vein thrombosis $(1.2 \%$ vs $0.37 \%$; OR, 3.3; $95 \% \mathrm{Cl}, 1.1-10.1 ; P=0.039)$, venous thromboembolism (1.9\% vs $0.74 \%$; OR, $2.7 ; 95 \% \mathrm{Cl}, 1.1-6.3, P=0.027$ ), serious complications (5.8\% vs $3.8 \%$; OR, $1.6 ; 95 \% \mathrm{Cl}, 1.0-2.4$; $P=0.031)$, permanently disabling complications $(1.2 \%$ vs $0.37 \%$; OR, 4.3; 95\% Cl, 1.2-15.6; $P=0.028)$, and death (0.7\% vs $0.09 \%$; OR, $7.0 ; 95 \% \mathrm{Cl}, 0.9-57.3 ; P=0.068$ ). Of the 7 deaths among patients with IVC filters, 4 were attributable to pulmonary embolism and 2 to IVC thrombosis/occlusion.

CONCLUSIONS: We have identified no benefits and significant risks to the use of prophylactic IVC filters among bariatric surgery patients and believe that their use should be discouraged. Journal of Hospital Medicine 2013;8:173-177. (C) 2013 Society of Hospital Medicine adverse events in patients receiving them. The United States Food and Drug Administration (FDA) has received more than 900 adverse event reports involving IVC filters, prompting the agency to issue a warning about their use. ${ }^{2}$ A prior study by our group demonstrated a lack of benefit of IVC filter insertion for the prevention of pulmonary embolism among bariatric surgery patients but lacked statistical power to prove harms associated with this practice. ${ }^{3}$

In the current study, we analyzed data from the prospective, statewide, clinical registry of the Michigan Bariatric Surgery Collaborative. Our study population now includes 35,477 bariatric surgery patients from 32 hospitals whose procedures were performed between 2006 and 2012. Since the publication of our prior study, the use of IVC filters in bariatric surgery has decreased significantly in Michigan. For this reason, our study population now includes many more highrisk patients who did not undergo IVC filter placement, allowing us to match IVC filter patients to similarly high-risk patients who did not receive IVC filters. We used these data to compare outcomes within 30 days of surgery, including rates of venous thromboembolism, overall serious complications, and death among patients who did and not receive IVC filters. 


\section{METHODS}

\section{Study Setting}

The Michigan Bariatric Surgery Collaborative (MBSC) is a regional voluntary consortium of hospitals and surgeons that perform bariatric surgery in Michigan. The goal of the project is to improve the quality of care for patients undergoing bariatric surgery. To do this, the participating hospitals submit data to the MBSC clinical outcomes registry, patient survey, and surgeon survey databases. Three times per year the group meets to examine these data and to design and implement changes in care to improve the outcomes of care for bariatric patients. The project is funded by Blue Cross and Blue Shield of Michigan/Blue Care Network and coordinated by faculty and staff members from the Center for Healthcare Outcomes and Policy at the University of Michigan.

The MBSC held its first collaborative meeting in June 2005 and enrolled its first patient in June 2006. The MBSC now has the participation of all of the 32 bariatric programs in Michigan, enrolling approximately 6000 patients per year in its clinical registry. Participating hospitals submit data from a review of the medical records for all of their bariatric surgery patients. This review is conducted for each patient at 30 days after surgery. The information collected includes preoperative clinical characteristics and conditions as well as perioperative clinical care and outcomes. The medical record reviews are performed by centrally trained, nurse data abstractors using a standardized and validated instrument. Each participating hospital is site visited annually to verify the accuracy and completeness of their MBSC clinical registry data.

\section{Study Population}

This study includes data for 35,477 patients undergoing bariatric surgery, including: 9829 laparoscopic adjustable gastric band, 6068 sleeve gastrectomy, 19,141 gastric bypass, and 439 biliopancreatic diversion with duodenal switch procedures between June 2006 and September 2012. Patients undergoing revisional bariatric surgery were excluded from these analyses. Prior to surgery, $1077(3.0 \%)$ of these patients had a prophylactic IVC filter placed for prevention of pulmonary embolism. Of the IVC filters placed, 39\% were temporary IVC filters, $45 \%$ were permanent IVC filters, and the type of IVC filter was not known in $15 \%$.

\section{Baseline Clinical Characteristics}

Data collected included patient demographic characteristics (age, gender, race, type of insurance), clinical characteristics (height, weight, history of cigarette smoking, mobility limitations), and obesity-related and other comorbid conditions (lung disease, cardiovascular disease, hyperlipidemia, gastroesophageal reflux disease, peptic ulcer disease, cholelithiasis, urinary incontinence, renal disease, diabetes, liver dis- ease, prior history of venous thromboembolism, sleep apnea, and psychological disorders).

Risk factors for VTE were empirically derived from our data base using multivariate statistical models. Risk factors for VTE included: age, body mass index, male sex, current or past smoking, mobility limitations, asthma, home oxygen use, peripheral vascular disease, prior history of VTE, bariatric procedure time, and procedure type. The baseline predicted risk for VTE was calculated for each patient based on these risk factors and was used to divide patients into low- (predicted risk $<1 \%$ ), medium- (predicted risk $1 \%-2.5 \%$ ), and high- (predicted risk $\geq 2.5 \%$ ) risk groups. Among the 35,477 patients in the registry overall, $95 \%$ are in the low-risk group, $4 \%$ are in the medium-risk group, and $1 \%$ are in the high-risk group. In the matched study cohorts, $69 \%, 22 \%$, and $9 \%$ were in the high-, medium-, and low-risk groups, respectively.

\section{Medical Venous Thromboembolism Prophylaxis}

Data were also collected regarding the type of medical venous thromboembolism prophylaxis (unfractionated vs low molecular weight heparin) used preoperatively, postoperatively, and whether the patient was discharged to home on low molecular weight heparin.

\section{Outcomes}

Our primary outcome measures included postoperative venous thromboembolism (deep vein thrombosis or pulmonary embolism requiring treatment). We also assessed overall rate of complications and complications according to severity as follows: non-life threatening complications (surgical site infection including wound and port site infections treated with antibiotics and/or wound opening, anastomotic stricture requiring dilatation, bleeding requiring blood transfusion of $<4$ units, and pneumonia requiring treatment with antibiotics only); potentially life-threatening complications (abdominal abscess requiring percutaneous drainage or reoperation, bowel obstruction requiring reoperation, leak requiring percutaneous drainage or reoperation, bleeding requiring transfusion $>4$ units, reoperation, or splenectomy, band-related problems requiring reoperation, respiratory failure requiring 2 to 7 days intubation, renal failure requiring in-hospital dialysis, wound infection/dehiscence requiring reoperation, and venous thromboembolism); and life-threatening complications associated with residual and lasting disability or death (myocardial infarction or cardiac arrest, renal failure requiring long-term dialysis, respiratory failure requiring $>7$ days intubation or tracheostomy, and death). Other complications that are not included in these categories (eg, IVC filter related) were assessed by an end points committee to determine their severity (non-life threatening, potentially life threatening, or life threatening associated with residual and lasting disability or death). 


\begin{tabular}{|c|c|c|c|c|c|}
\hline Variable & $\begin{array}{l}\text { IVC } \\
\text { Filter }\end{array}$ & $\begin{array}{l}\text { Matched } \\
\text { Controls }\end{array}$ & P Value & $\begin{array}{l}\text { Unmatched } \\
\text { Controls }\end{array}$ & $P$ Value \\
\hline NNo. & 1077 & 1077 & & 33,323 & \\
\hline Age (mean, y) & 48 & 49 & 0.295 & 46 & $<0.0001$ \\
\hline Body mass index (mean, $\mathrm{kg} / \mathrm{m}^{2}$ ) & 58 & 57 & 0.061 & 47 & $<0.0001$ \\
\hline Male gender (\%) & 32 & 31 & 0.546 & 21 & $<0.0001$ \\
\hline Black race $(\%)$ & 27 & 25 & 0.667 & 15 & $<0.0001$ \\
\hline Private Insurance (\%) & 62 & 64 & 0.305 & 74 & $<0.0001$ \\
\hline Smoking in past year (\%) & 2 & 2 & 0.883 & 2 & 0.440 \\
\hline Mobility limitations (\%) & 18 & 18 & 0.780 & 5 & $<0.0001$ \\
\hline Lung dsease (\%) & 43 & 43 & 1.000 & 25 & $<0.0001$ \\
\hline Cardiovascular disease (\%) & 21 & 21 & 0.874 & 10 & $<0.0001$ \\
\hline Hypertension (\%) & 72 & 72 & 0.737 & 53 & $<0.0001$ \\
\hline Hyperlipidemia (\%) & 59 & 59 & 0.930 & 50 & $<0.0001$ \\
\hline GERD (\%) & 50 & 52 & 0.490 & 49 & 0.417 \\
\hline Peptic ulcer disease (\%) & 5 & 4 & 0.228 & 3 & $<0.0001$ \\
\hline Cholelithiasis (\%) & 30 & 30 & 0.963 & 27 & 0.018 \\
\hline Urinary incontinence (\%) & 25 & 25 & 0.960 & 22 & 0.029 \\
\hline Renal failure (\%) & 0.4 & 0.6 & 0.526 & 0.2 & 0.298 \\
\hline Diabetes (\%) & 46 & 48 & 0.546 & 33 & $<0.0001$ \\
\hline Liver disorder (\%) & 4 & 4 & 0.584 & 5 & 0.184 \\
\hline Prior history of VTE (\%) & 39 & 39 & 0.965 & 2 & $<0.0001$ \\
\hline Sleep apnea (\%) & 70 & 68 & 0.209 & 43 & $<0.0001$ \\
\hline Musculoskeletal disorder (\%) & 78 & 80 & 0.221 & 77 & 0.189 \\
\hline History of hernia repair (\%) & 5 & 6 & 0.924 & 3 & $<0.0001$ \\
\hline Psychological disorder (\%) & 49 & 49 & 0.796 & 47 & 0.267 \\
\hline Total comorbidities (mean, no.) & 6 & 6 & 0.922 & 4 & $<0.0001$ \\
\hline \multicolumn{6}{|l|}{ Procedure } \\
\hline Adjustable gastric banding (\%) & 15 & 17 & 0.099 & 29 & $<0.0001$ \\
\hline Sleeve gastrectomy (\%) & 12 & 13 & 0.515 & 17 & $<0.0001$ \\
\hline Gastric bypass (\%) & 73 & 69 & 0.058 & 53 & $<0.0001$ \\
\hline Duodenal switch (\%) & 0.7 & 0.8 & 0.616 & 1.3 & $<0.0001$ \\
\hline Procedure Length (mean, minutes) & 114 & 116 & 0.427 & 95 & $<0.0001$ \\
\hline \multicolumn{6}{|l|}{ Medical VTE prophylaxis } \\
\hline \multicolumn{6}{|l|}{ Preoperative heparin: } \\
\hline Unfractionated (\%) & 36 & 38 & 0.246 & 34 & 0.306 \\
\hline Low molecular weight $(\%)$ & 60 & 54 & 0.017 & 53 & $<0.0001$ \\
\hline \multicolumn{6}{|l|}{ Postoperative heparin: } \\
\hline Unfractionated (\%) & 7 & 10 & 0.023 & 19 & $<0.0001$ \\
\hline Low molecular weight (\%) & 70 & 68 & 0.326 & 64 & $<0.0001$ \\
\hline \multicolumn{6}{|l|}{ Postdischarge heparin: } \\
\hline Low molecular weight $(\%)$ & 72 & 66 & 0.003 & 16 & $<0.0001$ \\
\hline
\end{tabular}

NOTE: Abbreviations: GERD, gastroesophageal reflux disease; IVC, inferior vena cava; VTE, venous thromboembolism.

\section{Statistical Analyses}

We used propensity score matching to assemble cohorts in which patients with and without IVC filters were balanced on baseline characteristics. The probability of IVC filter placement was estimated for each patient using a nonparsimonious multivariate logistic regression model, in which IVC filter was the dependent variable and all of the demographic, weight, medical history, weight-related comorbidity, and procedure-related variables (type, length, and year of procedure; and medical venous thromboembolism prophylaxis used) in our dataset were included as covariates. IVC filter patients were matched to control patients using a greedy, 1- to -1 matching without replacement protocol resulting in cohorts that were well balanced on all baseline characteristics.
TABLE 2. Relationship Between Inferior Vena Cava

Filter Use and Outcomes in Matched Analyses

\begin{tabular}{llll}
\hline Outcomes & Odds Ratio & $\begin{array}{l}95 \% \text { Confidence } \\
\text { Interval }\end{array}$ & $P$ Value \\
\hline Venous thromboembolism & 2.7 & $1.1-6.3$ & 0.027 \\
Deep vein thrombosis & 3.3 & $1.1-10.1$ & 0.039 \\
Pulmonary embolism & 2.0 & $0.6-6.5$ & 0.232 \\
$\quad$ Low-risk subgroup & 1.0 & $0.1-7.7$ & 0.965 \\
$\quad$ Medium-risk subgroup & 2.5 & $0.5-12.7$ & 0.288 \\
$\quad$ High-risk subgroup & 2.2 & $0.2-24.3$ & 0.530 \\
Any complication & 1.3 & $1.0-1.7$ & 0.048 \\
Serious complication & 1.6 & $1.0-2.4$ & 0.031 \\
Permanently disabling complication & 4.3 & $1.2-15.6$ & 0.028 \\
Death & 7.0 & $0.9-57.3$ & 0.068 \\
\hline
\end{tabular}

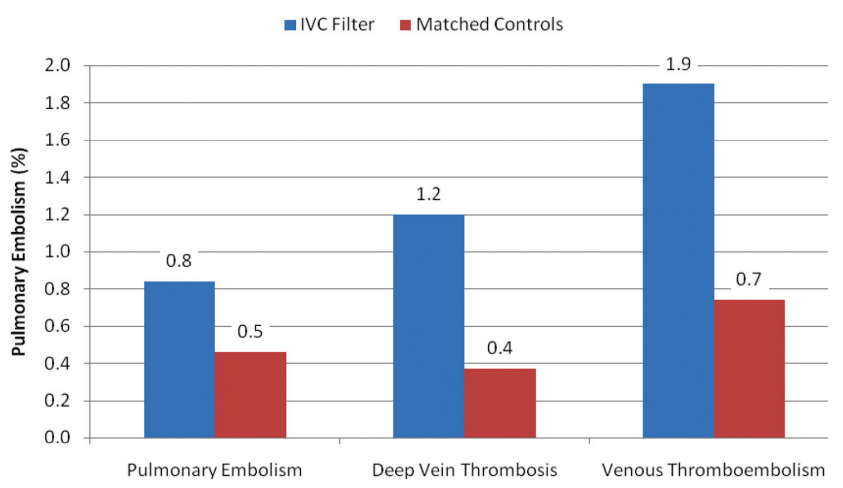

FIG. 1. Rates of pulmonary embolism, deep vein thrombosis, and venous thromboembolism in patients receiving prophylactic inferior vena cava (IVC) filters and in matched controls.

Baseline characteristics and outcomes were then compared among the cohorts using $\chi^{2}$ and $t$ tests as appropriate. We used mixed effects logistic regression to compare outcomes between the 2 treatment groups while controlling for clustering at the hospital and surgeon level as random effects. Odds ratios (OR) and 95\% confidence intervals (CI) were calculated to compare outcomes among patients with and without IVC filters.

\section{RESULTS}

Matching resulted in cohorts of IVC filter and control patients who were well balanced on all baseline characteristics (Table 1). In contrast, there were large and significant differences between IVC filter patients and unmatched control patients. For example, mean body mass index was 58 and 57 in the matched cohorts and 47 in the unmatched control patients. Prior history of venous thromboembolism was present in 39\%, $39 \%$, and $2 \%$ of the IVC filter, matched control, and unmatched control patients, respectively. With regard to procedure mix, unmatched control patients were less likely to have open gastric bypass and more likely to have adjustable gastric band procedures than IVC filter or matched control patients.

With regard to outcomes (Table 2, Figures 1 and 2), IVC filter patients had significantly higher rates of venous thromboembolism ( $1.9 \%$ vs $0.74 \%$; OR, 2.7 ; 


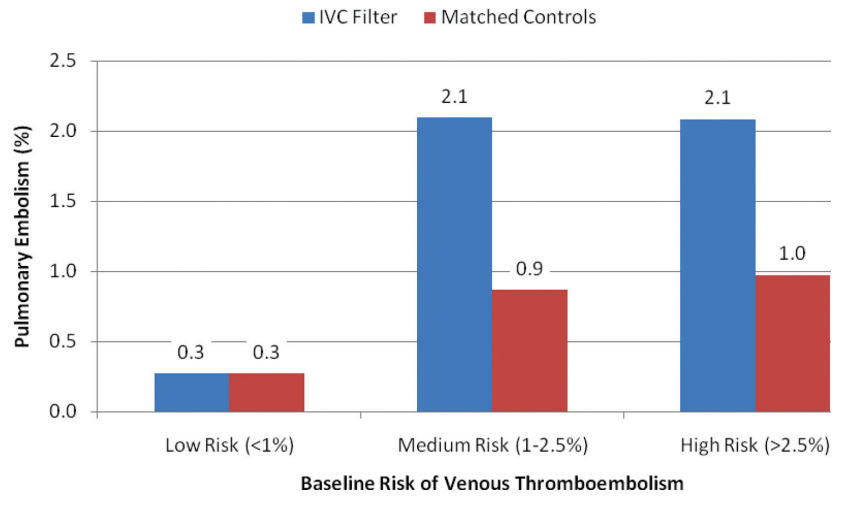

FIG. 2. Rates of pulmonary embolism in patients receiving prophylactic inferior vena cava (IVC) filters and in matched control patients according to baseline risk of venous thromboembolism.

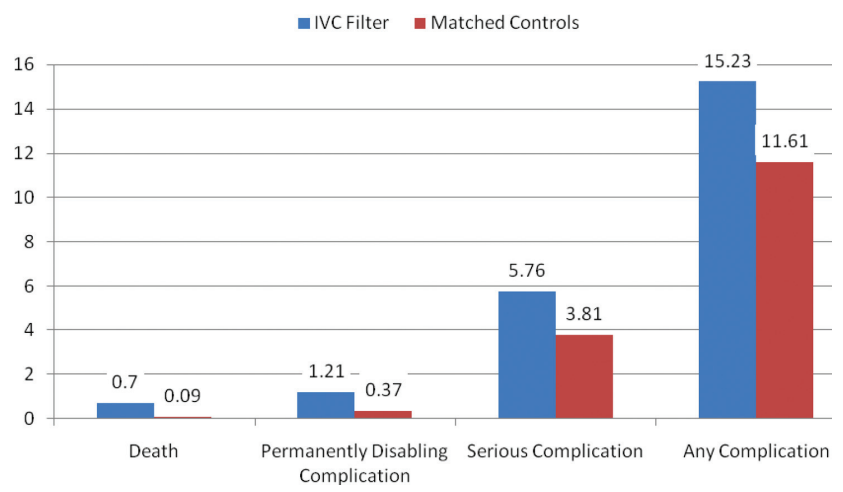

FIG. 3. Rates of complications according to severity in patients receiving prophylactic inferior vena cava (IVC) filters and in matched controls.

95\% CI, 1.1-6.3; $P=0.027)$ and deep vein thrombosis $(1.2 \%$ vs $0.37 \%$, OR, $3.3 ; \quad 95 \%$ CI, 1.1-10.1; $P=0.039)$ than matched control patients. Rates of pulmonary embolism were higher among IVC filter patients, but the difference was not statistically significant $(0.84 \%$ vs $0.46 \%$; OR, $2.0 ; 95 \%$ CI, 0.6-6.5; $P=0.232$ ). Rates of pulmonary embolism were similar for patients with a low baseline risk of venous thromboembolism $(0.27 \%$ vs $0.27 \%$; OR, $1.0 ; 95 \% \mathrm{CI}$, $0.1-7.7 ; P=0.965)$ but were higher for medium-risk patients $(2.1 \%$ vs $0.87 \%$; OR, 2.5 ; $95 \%$ CI, 0.5-12.7; $P=0.288)$ and high-risk patients $(2.1 \%$ vs $0.97 \%$; OR, 2.2; 95\% CI, 0.2-24.3; $P=0.530$ ).

Rates of other complications were also higher among IVC filter than matched control patients (Table 2 and Figure 3). There were significantly higher rates of complications $(15.2 \%$ vs $11.6 \%$; OR, $1.3 ; 95 \% \mathrm{CI}$, $1.0-1.7 ; \quad P=0.048)$, serious complications $(5.8 \%$ vs $3.8 \%$; OR, $1.6 ; 95 \%$ CI, $1.0-2.4 ; P=0.031)$, and permanently disabling complications $(1.2 \%$ vs $0.4 \%$; OR, 4.3; 95\% CI, 1.2-15.6; $P=0.028)$ among IVC filter patients. Rates of death $(0.7 \%$ vs $0.1 \%$; OR, 7.0 ; 95\% CI, 0.9-57.3; $P=0.068)$ were also higher among IVC filter patients than matched control patients, but this difference was not statistically significant.

Among the 7 IVC filter patients who died, 4 had fatal pulmonary embolism, and 2 had IVC filter thrombosis/occlusion. Other IVC filter-specific complications included IVC filter migration requiring heart valve replacement surgery in 1 patient, contrastinduced nephropathy in 1 patient, IVC filter incision site infection in 1 patient, and technical difficulties removing a temporary IVC filter requiring that the device stay in place in 1 patient.

\section{DISCUSSION}

In this propensity matched, observational cohort study, we assessed the safety and effectiveness of prophylactic IVC filters among bariatric surgery patients. We found that patients with IVC filters had significantly worse outcomes than comparably highrisk patients without IVC filters. Rates of venous thromboembolism were higher in the IVC filter patients, and a large proportion of the other complications among IVC filter patients were device related.

Our current study of IVC filters was prompted by an FDA advisory report regarding complications in patients receiving IVC filters. ${ }^{2}$ The FDA's report was in turn prompted by a study indicating a high prevalence of strut fracture and embolization among 80 patients who received a certain type of retrievable IVC filter. ${ }^{4}$ The FDA conveyed receiving 921 adverse events reports involving IVC filters between 2005 and 2009. Thirty-six percent of these reports involved migration of the device, $16 \%$ were related to breakage and embolization of parts of the device, and $8 \%$ involved perforation of the IVC.

Research on the safety and efficacy of IVC filters in bariatric surgery patients has largely been limited to small, single-center, case series or cohort studies. ${ }^{5-15}$ A systematic review of this literature concluded that the evidence was insufficient to recommend IVC filters for patients undergoing bariatric surgery. ${ }^{16}$ A 2010 study by our group was the largest and only multicenter study of IVC filters in the bariatric surgery population. We found no benefit of IVC filters in a comparison of 542 gastric bypass patients with prophylactic IVC filters to 5,834 gastric bypass patients without prophylactic IVC filters. ${ }^{3}$

When interpreting the results of this study, a number of limitations should be considered. Our study was observational, so there is the potential for unmeasured confounding variables to have influenced our results. To minimize the risk of confounding, we used propensity scores to match IVC filter patients to comparably high-risk control patients, resulting in study cohorts that were well balanced on all baseline variables. Although this method accounts for confounding on the variables for which there are data, there is still the possibility that an unknown confounder could affect our findings. For example, our clinical registry lacks data on hypercoagulable states, so it is possible that a higher proportion of IVC filter patients could have had this risk factor and therefore 
a higher baseline risk of venous thromboembolism. However, most patients with a hypercoagulable state would have had a prior history of venous thromboembolism, which is a variable included in our database that patients were matched on.

The effects of changes in clinical care occurring during the time frame of this study should be considered in interpreting our findings. For example, bariatric surgery has been getting safer in general over time. Rates of death have fallen both in Michigan and in the rest of the country as bariatric surgeons have gained experience with this procedure. In Michigan during this time period, our group has developed and implemented a risk-stratified, standardized approach to venous thromboembolism prophylaxis for patients undergoing bariatric surgery. For these reasons, we included the year of the procedure and the type of medical venous thromboembolism prophylaxis (unfractionated or low molecular weight heparin) used perioperatively as a matching variable in our analysis.

Another limitation that should be considered in interpreting our findings is statistical power. Although our study is the largest in this study population to date, many of the outcomes of interest are relatively rare. Considering the entire bariatric surgery population, rates of venous thromboembolism and death within 30 days are each less than $1 \%$. Even in the high-risk patients included in this analysis, there were a total of just $28(1.3 \%)$ venous thromboembolism events and 8 (0.37) deaths. Nonetheless, our study did find significantly greater risks of multiple types of complications among patients receiving IVC filters.

Finally, our study captures events occurring within 30 days of bariatric surgery. Complications, including venous thromboembolism and other complications directly related to IVC filters, frequently occur after 30 days of bariatric surgery. Therefore, our study may be a conservative estimate of the risks associated with the use of IVC filters in bariatric surgery patients. Furthermore, certain brands of filters have been shown to be associated with higher risks of complications. Our study lacks data on the brand of IVC filter used and so cannot assess the extent to which this would affect our results.

\section{CONCLUSIONS}

In conclusion, our study indicates that IVC filters do not reduce the risk of pulmonary embolism in high- risk bariatric surgery patients. They are also associated with other complications attributable to malfunctions of the device itself. We believe that the use of IVC filters among bariatric surgery patients should be discouraged.

Disclosures: This study was supported by a grant from the Agency for Healthcare Research and Quality (HS018050) and was presented at the Annual Meeting of the American Society for Metabolic and Bariatric Surgery (ASMBS), San Diego, California, June 20, 2012.

\section{References}

1. Streiff M, Kim K, Hong K. Vena cava filters: a call to action. Chest Physician. 2011;16:18a.

2. U.S. Food and Drug Administration. Removing retrievable inferior vena cava filters: initial communication. August 9, 2010. Available at: http://www.fda.gov/MedicalDevices/Safety/AlertsandNotices/ucm221 676.htm. Accessed December 2, 2012.

3. Birkmeyer N, Share D, Baser O, et al. Preoperative placement of inferior vena cava filters and outcomes after gastric bypass surgery. Ann Surg. 2010;252:131-318.

4. Nicholson W, Nicholson W, Tolerico P, et al. Prevalence of fracture and fragment embolization of Bard retrievable vena cava filters and clinical implications including cardiac perforation and tamponade. Arch Intern Med. 2010;170:1827-1831.

5. Gargiulo N, Veith F, Lipsitz E, Suggs W, Ohki T, Goodman E. Experience with inferior vena cava filter placement in patients undergoing open gastric bypass procedures. Ann Vasc Surg. 2006;44: 1301-1305.

6. Halmi D, Kolesnikov E. Preoperative placement of retrievable inferior vena cava filters in bariatric surgery. Surg Obes Relat Dis. 2007;3: 602-605.

7. Kardys C, Stoner M, Manwaring M, et al. Safety and efficacy of intravascular ultrasound-guided inferior vena cava filter in super obese bariatric patients. Surg Obes Relat Dis. 2008;4:50-54.

8. Keeling W, Haines K, Stone P, Armstrong P, Murr M, Shames M. Current indications for preoperative inferior vena cava filter insertion in patients undergoing surgery for morbid obesity. Obes Surg. 2005;15:1009-1012.

9. Obeid F, Bowling W, Fike J, Durant J. Efficacy of prophylactic inferior vena cava filter placement in bariatric surgery. Surg Obes Relat Dis. 2007;3:606-610.

10. Piano G, Ketteler E, Prachand V, et al. Safety, feasibility, and outcome of retrievable vena cava filters in high-risk surgical patients. $J$ Vasc Surg. 2007;45:784-788.

11. Schuster R, Hagedorn J, Curet M, Mortaon J. Retrievable inferior vena cava filters may be safely applied in gastric bypass surgery. Surg Endosc. 2007;21:2277-2279.

12. Trigilio-Black C, Ringley C, McBride C, Sorensen V, Thompson J. Inferior vena cava filter placement for pulmonary embolism risk reduction in super morbidly obese undergoing bariatric surgery. Surg Obes Relat Dis. 2007;3:461-464.

13. Frezza E, Wachtel M. A simple venous thromboembolism prophylaxis protocol for patients undergoing bariatric surgery. Obesity (Silver Spring). 2006;14:1961-1965.

14. Overby D, Kohn G, Cahan M, et al. Risk-group targeted inferior vena cava filter placemetn in gastric bypass patients. Obes Surg. 2009;19:451-455.

15. Vaziri K, Bhanot P, Hungness E, Morasch M, Prystowsky J, Nagle A. Retreivable inferior vena cava filters in high-risk patients undergoing bariatric surgery. Surg Endosc. 2009;23:2203-2207.

16. Rajasekhar A, Crowther M. Inferior vena caval filter insertion prior to bariatric surgery: A systematic review of the literature. I Thromb Haemost. 2010;8:1266-1270. 